\title{
Optimized Conformal Parameterization of Cortical Surfaces Using Shape Based Matching of Landmark Curves
}

\author{
Lok Ming Lui ${ }^{1}$, Sheshadri Thiruvenkadam ${ }^{1}$, Yalin Wang ${ }^{1,2}$, Tony Chan ${ }^{1}$, \\ and Paul Thompson ${ }^{2}$ \\ ${ }^{1}$ Department of Mathematics, UCLA, Los Angeles, CA 90095-1555 \\ ${ }^{2}$ Laboratory of Neuro Imaging and Brain Research Institute, UCLA School of \\ Medicine, CA 90095-1555
}

\begin{abstract}
In this work, we find meaningful parameterizations of cortical surfaces utilizing prior anatomical information in the form of anatomical landmarks (sulci curves) on the surfaces. Specifically we generate close to conformal parametrizations that also give a shape-based correspondence between the landmark curves. We propose a variational energy that measures the harmonic energy of the parameterization maps, and the shape dissimilarity between mapped points on the landmark curves. The novelty is that the computed maps are guaranteed to give a shapebased diffeomorphism between the landmark curves. We achieve this by intrinsically modelling our search space of maps as flows of smooth vector fields that do not flow across the landmark curves, and by using the local surface geometry on the curves to define a shape measure. Such parameterizations ensure consistent correspondence between anatomical features, ensuring correct averaging and comparison of data across subjects. The utility of our model is demonstrated in experiments on cortical surfaces with landmarks delineated, which show that our computed maps give a shape-based alignment of the sulcal curves without significantly impairing conformality.
\end{abstract}

\section{Introduction}

Parametrization of the cortical surface is a key problem in brain mapping research. Applications include the registration of functional activation data across subjects, statistical shape analysis, morphometry, and processing of signals on brain surfaces (e.g., denoising or filtering). Applications that compare surface data often make use of surface diffeomorphisms that result from parameterization. For the above diffeomorphisms to map data consistently across surfaces, parametrizations are required that preserves the original surface geometry as much as possible. Parameterizations should also be chosen so that the resulting diffeomorphisms between surfaces align key anatomical features consistently.

Conformal mapping [1,2] is particularly convenient for genus-zero cortical surface models since it gives a parameterization without angular distortions, and comes with computational advantages when solving PDEs on surfaces using

D. Metaxas et al. (Eds.): MICCAI 2008, Part I, LNCS 5241, pp. $4 9 4 \longdiv { 5 0 1 } 2 0 0 8 .$

(C) Springer-Verlag Berlin Heidelberg 2008 
grid-based and metric-based computations [3]. However, the above parameterization is not guaranteed to map anatomical features, such as sulcal landmarks, consistently from subject to subject [24].

Landmark-based diffeomorphisms 4567/89] are often used to compute, or adjust, cortical surface parameterizations. Similarly to the above works, given two cortical surfaces with anatomical landmarks (sulci curves), we want to find close to conformal parameterizations for the surfaces driven by shape based correspondences (registration) between the curves. Our work has three main contributions; first, the surface diffeomorphism resulting from our parameterization maps the sulcal curves exactly; second, the correspondence is shape based, i.e., maps similarly-shaped segments of sulcal curves to each other; finally, the conformality of the surface parameterizations is preserved to the greatest possible extent.

Optimization of surface diffeomorphisms by landmark matching has been studied intensively. Gu et al. 2] optimized the conformal parametrization by composing an optimal Möbius transformation so that it minimizes a landmark mismatch energy. The resulting parameterization remains conformal. Glaunes et al. 6] proposed to generate large deformation diffeomorphisms of the sphere onto itself, given the displacements of a finite set of template landmarks. The diffeomorphism obtained can match landmark features well, but it is, in general, not a conformal mapping, which can be advantageous for solving PDEs on the resulting grids. Leow et al. [7] proposed a level-set based approach for matching different types of features, including points and 2D or 3D curves represented as implicit functions.

Tosun et al. 8] proposed a more automated mapping technique that results in good sulcal alignment across subjects, by combining parametric relaxation, iterative closest point registration and inverse stereographic projection. Wang et al. 4 proposed an energy that computes maps that are close to conformal and also driven by a landmark matching term that measures the Euclidean distance between the specified landmarks.

Many of the above methods e.g. 46] require corresponding landmark points on the surfaces to be labeled in advance. Secondly, the landmark match measures used above are based on Euclidean distance, or overlap of level set functions representing the landmarks, and do not use shape information to guide correspondences of features within curves. So, the resulting correspondences would be unreliable in the case of landmark curves that differ by non-rigid deformations. Finally, constraining the surface diffeomorphism to exactly align the landmark curves during minimization is difficult, e.g. 48].

To resolve the above issues, we propose a method to optimize the conformal parameterization of the surfaces while non-rigidly registering the landmark curves. Specifically, we formulate our problem as a variational energy defined on a search space of diffeomorphisms generated as flows of smooth vector fields. The vector fields are restricted only to those that do not flow across the landmark curves (to enforce exact landmark correspondence). Our energy has 2 terms: (1) a shape term to map similar shaped segments of the landmark curves to each other, and (2) a harmonic energy term to optimize the conformality of the parametrization maps. 


\section{Model}

Given two cortical surfaces $M_{1}$ and $M_{2}$, with sulcal landmark curves $\hat{C}_{1}$ and $\hat{C}_{2}$ labeled on them. The curves $\hat{C}_{i}$ have the same topology relative to $M_{i}$. These landmarks curves can be detected automatically by the automatic landmark tracking technique introduced by Lui et al. [10. Here, we want to find diffeomorphisms $\hat{f}_{1}: \Omega \subset \Re^{2} \rightarrow M_{1}, \hat{f}_{2}: \Omega \rightarrow M_{2}$ such that $\hat{f}_{2}$ o $\left.\hat{f}_{1}^{-1}\right|_{\hat{C}_{1}}$ is a shape based diffeomorphism onto $\hat{C}_{2}$, i.e $\hat{f}_{2}$ o $\hat{f}_{1}^{-1}$ maps similarly shaped segments of $\hat{C}_{1}$ and $\hat{C}_{2}$ to each other. Also we want $\hat{f}_{i}$ to be as conformal as possible.

To simplify our computations, $M_{i}$ are firstly conformally parameterized onto the conformal parameter domain $D_{i}$. Assume that $\hat{C}_{i}$ are mapped to $C_{i}$ on the parameter domain $D_{i}$. Thus, our problem is reduced to the $2 \mathrm{D}$ problem of finding diffeomorphism $\tilde{f}_{i}: \Omega \rightarrow D_{i}$ such that $\tilde{f}_{2}$ o $\left.\tilde{f}_{1}^{-1}\right|_{C_{1}}=C_{2}$ is a shapebased diffeomorphism onto $C_{2}$. We propose our problem as the minimization of a variational energy with respect to diffeomorphisms $\tilde{f}_{i}: \Omega \rightarrow D_{i}$, subject to the correspondence constraint $\tilde{f}_{2}$ o $\tilde{f}_{1}^{-1}\left(C_{1}\right)=C_{2}$. The energy consists of two terms. The first term measures the harmonic energy of the maps $\tilde{f}_{i}$, and the second term measures the shape dissimilarity between $C_{1}$ and $\tilde{f}_{2}$ o $\tilde{f}_{1}^{-1}\left(C_{1}\right)$.

To handle the above correspondence constraint, we move all our computations to the parameter domain $\Omega$ using initial diffeomorphisms $f_{0, i}: \Omega \rightarrow D_{i}$. Let $C \subset \Omega$ be a topological representative of $C_{i}$, with $f_{0, i}(C)=C_{i}$. With the above framework, the energy is formulated over $\Omega$, and the search space of diffeomorphisms $\tilde{f}_{i}: \Omega \rightarrow D_{i}$, subject to $\tilde{f}_{2}$ o $\tilde{f}_{1}^{-1}\left(C_{1}\right)=C_{2}$, can be constructed as time-1 flows of smooth vector fields on $\Omega$ that do not flow across $C$. For the shape term, we measure the shape dissimilarity between the corresponding landmarks which minimizes the difference in geodesic curvatures on the corresponding pairs of points on $C_{1}$ and $C_{2}$. We discuss the details in the following sections.

\subsection{Formulation}

The initial diffeomorphisms $f_{0, i}$ give us a convenient way to perform our computations on the domain $\Omega$. Diffeomorphisms $\tilde{f}_{i}: \Omega \rightarrow D_{i}$ with $\tilde{f}_{2}$ o $\tilde{f}_{1}^{-1}\left(C_{1}\right)=C_{2}$ can be realized through unique diffeomorphisms $f_{i}: \Omega \rightarrow \Omega$ with $f_{i}(C)=C$, satisfying $\tilde{f}_{i}=f_{0, i}$ o $f_{i}$ (Fig. 1)(left)). Thus we formulate our problem as the minimization of the following energy over diffeomorphisms $f_{i}: \Omega \rightarrow \Omega$ with $f_{i}(C)=C$. Denote $\tilde{f}_{i}=f_{0, i}$ o $f_{i}, F=\left[\tilde{f}_{1}, \tilde{f}_{2}\right]$,

$$
E\left[f_{1}, f_{2}\right]=\int_{\Omega}\left|\nabla \tilde{f}_{1}\right|^{2}+\left|\nabla \tilde{f}_{2}\right|^{2} d x+\lambda \int_{C}\left(\kappa_{1}\left(\tilde{f}_{1}\right)-\kappa_{2}\left(\tilde{f}_{2}\right)\right)^{2}\left|F_{x} \wedge F_{y}\right| d s
$$

The first term is the harmonic energy of $\tilde{f}_{i}$. The second term is a symmetric shape term defined as an arc length integral over $F(C)$, similar to Thiruvenkadam et al. [11. Here, the shape measure $\kappa_{i}\left(p_{i}\right)$ is determined by the geodesic curvature of $M_{i}$ corresponding to the point $p_{i}$. Defining the symmetric shape measure over $F(C)$ makes the term independent of the choice of the initial maps $f_{0, i}$, and also avoids local minima problems that occur while matching flat curve segments. 

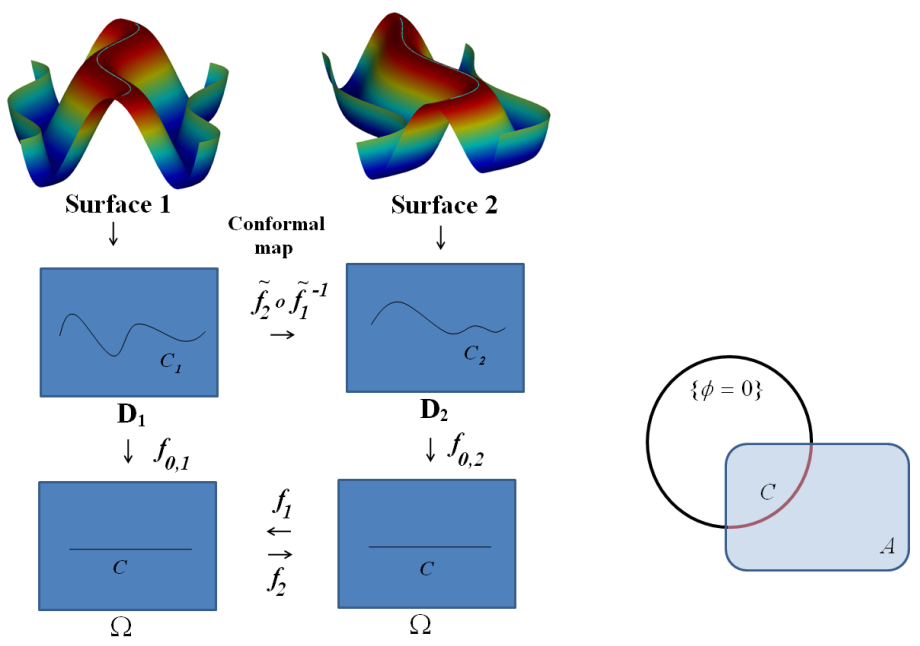

Fig. 1. The left panels show the framework of our algorithm. The right panel shows the level set representation for $C$ (Brown open curve), $C=\{\phi=0\} \cap A$. $A$ is the shaded region, $\{\phi=0\}$ is the circle.

In the above energy, using a search space of diffeomorphisms $f_{i}: \Omega \rightarrow \Omega$, and then imposing $f_{i}(C)=C$ as a constraint during minimization is difficult. Hence we propose a method to directly consider a reduced search space of diffeomorphisms $f_{i}: \Omega \rightarrow \Omega$ that satisfy $f_{i}(C)=C$.

\subsection{Level Set Representation for C}

Since we are dealing with the sulcal curves as our landmarks, we assume that $C=\cup_{k=1}^{N} \Gamma_{k}$, a union of open curves $\Gamma_{k} \subset \Omega$. We represent $C$ implicitly in level set form to be able to write the second integral in energy (1) with respect to $x$. Being the union of open curves, $C$ can be represented as the intersection of the 0 -level set of a signed distance function $\phi$, and a region $A$ (Fig. 11(right)). Then the arc length integral of $C$ becomes

$$
\int_{C} d s=\int_{\Omega} \chi_{A}|\nabla H(\phi)| d x
$$

where $H(t)$ is a regularized version of the Heaviside function.

\subsection{Modelling the Search Space for $f_{i}$}

To construct an appropriate search space for $f_{i}$, we consider smooth vector fields, $\boldsymbol{X}_{\boldsymbol{i}}=a_{i} \frac{\partial}{\partial x}+b_{i} \frac{\partial}{\partial y}$, where $a_{i}, b_{i}: \Omega \rightarrow \Re$ are $C^{1}$ functions with compact support. Then the flow of $\boldsymbol{X}_{i}, \Phi^{\boldsymbol{X}_{i}}(\mathbf{x}, t)$ is given by the differential equation,

$$
\begin{array}{r}
\frac{\partial \Phi^{\boldsymbol{X}_{i}}}{\partial t}(\mathbf{x}, t)=\boldsymbol{X}_{i}\left(\Phi^{\boldsymbol{X}_{i}}(\mathbf{x}, t)\right), \\
\Phi^{\boldsymbol{X}_{i}}(\mathbf{x}, 0)=\mathbf{x} .
\end{array}
$$

Then the time-1 flow $\Phi^{\boldsymbol{X}_{i}}(\mathbf{x}, 1): \Omega \rightarrow \Omega$ is a diffeomorphism. 
Now let $\boldsymbol{n}:=\tilde{\delta}(\phi) \tilde{\chi}_{A} \nabla \phi$, for regularized versions $\tilde{\delta}, \tilde{\chi}_{A}$ of the Dirac- $\delta$ function, and $\chi_{A}$. We see that $\boldsymbol{n}$ coincides with the unit-normal vector field on $C$. Let $\eta_{e p}$ be a smooth function on $\Omega$ such that $\eta_{e p}=0$ at the endpoints of the open curves $\Gamma_{k} \subset C, k=1,2, . . N$. Consider the vector fields $\boldsymbol{Y}_{i}$ that do not flow across $C$,

$$
\boldsymbol{Y}_{i}=P_{C} X_{i}:=\eta_{e p}\left(\boldsymbol{X}_{i}-\left(\boldsymbol{X}_{i} \cdot \boldsymbol{n}\right) \boldsymbol{n}_{i}\right) .
$$

We notice the following properties for the time-1 flow, $\Phi^{\boldsymbol{Y}_{i}}(., 1)$,

$-\Phi^{\boldsymbol{Y}_{i}}(., 1): \Omega \rightarrow \Omega$ is a diffeomorphism since $\boldsymbol{Y}_{i}$ is $C^{1}$.

- Also $\left.\boldsymbol{Y}_{i}\right|_{C}$ is a $C^{1}$ vector field on $C$. Thus $\left.\Phi^{\boldsymbol{Y}_{i}}(., 1)\right|_{C}$ is a diffeomorphism onto $C$.

Hence it is natural to set $f_{i}=\Phi^{\boldsymbol{Y}_{i}}(., 1)$.

\subsection{Energy}

We formulate the energy (1) over the space of $C^{1}$ smooth vector fields on $\Omega$, $\boldsymbol{X}_{\boldsymbol{i}}=a_{i} \frac{\partial}{\partial x}+b_{i} \frac{\partial}{\partial y}$, $J\left[a_{i}, b_{i}\right]=$

$$
\begin{aligned}
\int_{\Omega}\left|\nabla \tilde{f}_{1}\right|^{2}+\left|\nabla \tilde{f}_{2}\right|^{2} d x+\lambda \int_{\Omega} \chi_{A}\left(\kappa_{1}\left(\tilde{f}_{1}\right)\right. & \left.-\kappa_{2}\left(\tilde{f}_{2}\right)\right)^{2}|\nabla H(\phi)|\left|F_{x} \wedge F_{y}\right| d x \\
& +\beta \int_{\Omega}\left|D \boldsymbol{X}_{1}\right|^{2}+\left|D \boldsymbol{X}_{2}\right|^{2} d x
\end{aligned}
$$

Here, as before $\tilde{f}_{i}=f_{0, i}$ o $f_{i}$, and $f_{i}=\Phi^{\boldsymbol{Y}_{i}}(., 1)$, the time-1 flow of the vector field $\boldsymbol{Y}_{i}=P_{C} X_{i}$. The last integral in the energy is the smoothness term for the vector fields $\boldsymbol{X}_{i}$. To minimize the above energy, we can iteratively modify the vector field $\boldsymbol{X}_{i}$ by the following Euler-Lagrange equation:

$$
\begin{aligned}
\frac{d a_{i}}{d t} & =\int_{0}^{1} B_{i}\left(\phi_{s}^{\boldsymbol{Y}_{i}}\right) \Psi_{i}\left(\phi_{s}^{\boldsymbol{Y}_{i}}, 1\right) \Psi_{i}^{-1}\left(\phi_{s}^{\boldsymbol{Y}_{i}}, s\right) P_{C} \boldsymbol{e}_{1}\left|D \phi_{s}^{\boldsymbol{Y}_{i}}\right| d s-\beta \Delta a_{i} \\
\frac{d b_{i}}{d t} & =\int_{0}^{1} B_{i}\left(\phi_{s}^{\boldsymbol{Y}_{i}}\right) \Psi_{i}\left(\phi_{s}^{\boldsymbol{Y}_{i}}, 1\right) \Psi_{i}^{-1}\left(\phi_{s}^{\boldsymbol{Y}_{i}}, s\right) P_{C} \boldsymbol{e}_{2}\left|D \phi_{s}^{\boldsymbol{Y}_{i}}\right| d s-\beta \Delta b_{i},
\end{aligned}
$$

where: $B_{i}:=-\Delta \tilde{f}_{i} D f_{0, i}+\lambda \chi_{A}\left((-1)^{i-1}\left(\kappa_{1}\left(\tilde{f}_{1}\right)-\kappa_{2}\left(\tilde{f}_{2}\right)\right) \nabla \kappa_{i}-\nabla \cdot C_{i}\right) D f_{0, i}|\nabla H(\phi)|$; $\Psi_{i}$ is the orthogonal fundamental matrix for the homogeneous system of

$$
\frac{\partial}{\partial t} P_{i}(x, t)=\eta P_{C} \boldsymbol{e}_{1}\left(\Phi^{\boldsymbol{Y}_{i}}(x, t)\right)+D \boldsymbol{Y}_{i}\left(\Phi^{\boldsymbol{Y}_{i}}(x, t)\right) P_{i}(x, t), P_{i}(x, 0)=\mathbf{0} .
$$

\section{Experimental Results}

We have tested our automatic landmark tracking algorithm on cortical hemispheric surfaces extracted from brain MRI scans, acquired from normal subjects at $1.5 \mathrm{~T}$ (on a GE Signa scanner). Experimental results show that our algorithm can effectively compute cortical surface parameterizations that align the landmark features in a way that also enforces shape correspondence, while preserving 


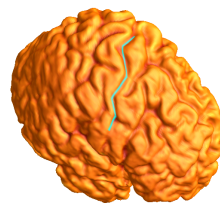

Brain 1

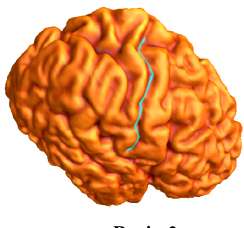

Brain 2

Fig. 2. The figure shows two different cortical surfaces with sulcal landmarks

the conformality of the surface-to-surface mapping to the greatest extent possible. The computed map is guaranteed to be a diffeomorphism because the map is formulated as the integral flow of a smooth vector field.

Figure 2 shows two different cortical surfaces with sulcal landmarks labeled on them. We seek parameterizations of these surfaces that align the landmark features consistently while optimally preserving conformality. A diffeomorphism between the two surfaces is then obtained by computing the composition of the two parameterizations. Figure 3 shows the result of matching the cortical surfaces with one landmark labeled (for purposes of illustration) on each brain. Figure 3(A) shows the cortical surface of Brain 1. It is mapped to the cortical surface of Brain 2 under the conformal parameterization as shown in Figure 3(B). Note that the sulcal landmark on Brain 1 is only mapped approximately to the sulcal region on Brain 2. It is not mapped exactly to the corresponding sulcal landmark on Brain 2. Figure 3(C) shows the matching result under the parameterization we propose in this paper. Note that the corresponding landmarks are mapped exactly. Also, the correspondence between the landmark curves follows the shape information.

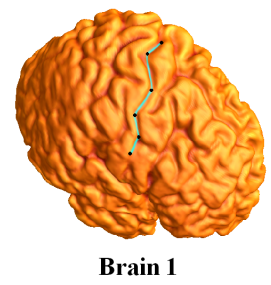

(A)

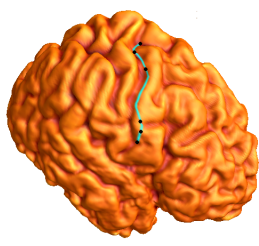

Brain 2

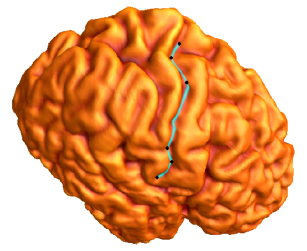

Brain 2

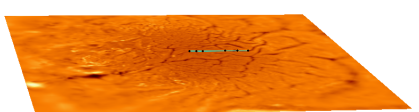

2D domain (Brain 1)

(D)
(B)

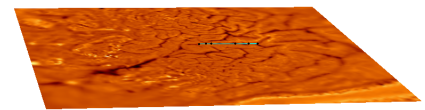

2D domain (Brain 2)

(E)

Fig. 3. This figure shows the result of matching the cortical surfaces with one landmark labeled. (A) shows the surface of Brain 1. It is mapped to Brain 2 under conformal parameterization, as shown in (B). (C) shows the result of matching using our proposed algorithm. (D) and (E) show the standard 2D parameter domains for Brain 1 and Brain 2 respectively. 


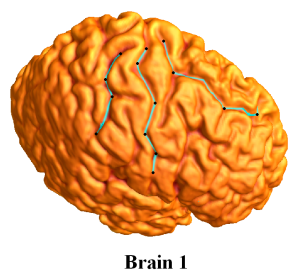

(A)

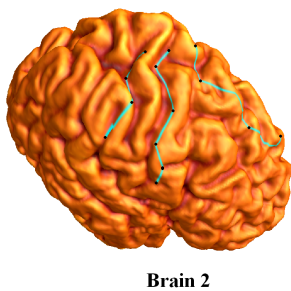

(B)

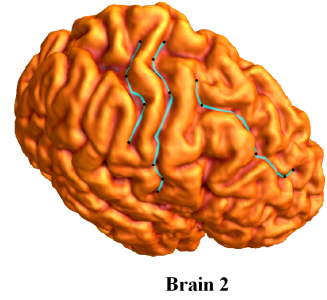

(C)

Fig. 4. Illustration of the result of matching the cortical surfaces with several sulcal landmarks. (A) shows the brain surface 1. It is mapped to brain surface 2 under the conformal parameterization as shown in (B). (C) shows the result of matching under our proposed parameterization.
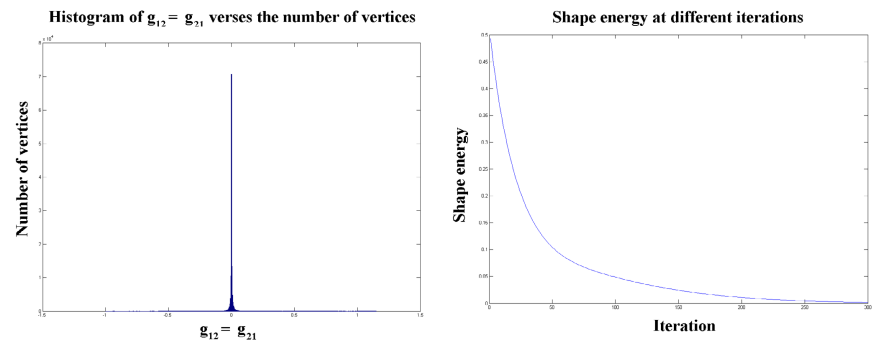

Fig. 5. The left shows the histogram of $g_{12}=g_{21}$ of the brain surface under the parameterization computed with our algorithm. The right shows the shape energy at different iterations.

It maps the secondary features of one landmark curve to the secondary features of the other landmark curve (See the black dots). Figure 3(D) and (E) show the standard 2D parameter domain of Brain 1 and Brain 2 respectively. The landmark curve is mapped to same horizontal line and the shape feature are mapped to the same positions (see the black dots). This is advantageous as the surface average of many subjects would retain features that consistently occur on sulci, while uniform speed parameterizations may cause these features to cancel out. Figure 4 gives an illustration of the matching results for cortical surfaces with several sulcal landmarks labeled on them. Figure 4(A) shows the brain surface 1 with several landmarks labeled. It is mapped to brain surface 2 under the conformal parameterization as shown in Figure 4(B). Again, the sulcal landmarks on Brain 1 are only mapped approximately to the sulcal regions on Brain 2. Figure 4 (C) shows the matching result under the parameterization we proposed. The corresponding landmarks are mapped exactly. Also, the correspondence between the landmark curves follows the shape information (corners to corners [See the black dot]). To examine the conformality of the parameterization, we show in Figure 5 ( Left) the histogram of $g_{12}=g_{21}$ of the Riemannian metric under the parameterization computed with our proposed algorithm. Observe that $g_{12}=g_{21}$ are very close to zero at most vertices. This means that the Riemannian metric is a diagonal matrix, 
thus the parameterization computed is very close to conformal. It also shows that conformal map being intrinsic to global surface geometry, is not significantly affected by small changes in the local geometry induced by the shape term. Figure $5($ Right) shows that the shape energy is decreasing with iterations, implying an improving shape based correspondence between the landmark curves.

\section{Conclusion and Future Work}

In this paper, we developed an algorithm to find parametrizations of the cortical surfaces that are close to conformal and also give a shape based correspondence between embedded landmark curves. We propose a variational approach by minimizing an energy that measures the harmonic energy of the parameterization maps, and the shape dissimilarity between mapped points on the landmark curves. The parameterizations computed are guaranteed to give a shape-based diffeomorphism between the landmark curves. Experimental results show that our algorithm can effectively compute parameterizations of cortical surfaces that align landmark features consistently with shape correspondence, while preserving the conformality as much as possible. As future work, we plan to apply this algorithm to cortical models from healthy and diseased subjects to build population shape averages. The enforcement of higher-order shape correspondences may allow subtle but systematic differences in cortical patterning to be detected, for instance in neurodevelopmental disorders such as Williams syndrome, where the scope of cortical folding anomalies is of great interest but currently unknown. Another area of interest is to work on better numerical schemes to improve computational efficiency and accuracy.

\section{References}

1. Haker, S., Angenent, S., Tannenbaum, A., Kikinis, R., Sapiro, G., Halle, M.: IEEE TVCG 6(2), 181-189 (2000)

2. Gu, X., Wang, Y., Chan, T.F., Thompson, P.M., Yau, S.T.: IEEE TMI 23(8), 949-958 (2004)

3. Lui, L., Wang, Y., Chan, T.F.: VLSM. In: ICCV (2005)

4. Wang, Y., Lui, L., Chan, T.F., Thompson, P.: In: Duncan, J.S., Gerig, G. (eds.) MICCAI 2005, Part I. LNCS, vol. 3750, pp. 675-683. Springer, Heidelberg (2005)

5. Gu, X., Yau, S.: ACM Symp. on Geom. Processing 2003 (2003)

6. Glaunés, J., Vaillant, M., Miller, M.: J. Maths. Imaging and Vision 20, 179-200 (2004)

7. Leow, A., Yu, C., Lee, S., Huang, S., Protas, H., Nicolson, R., Hayashi, K., Toga, A., Thompson, P.: NeuroImage 24(3), 910-927 (2005)

8. Tosun, D., Rettmann, M., Prince, J.: Med. Image Anal. 8(3), 295-309 (2004)

9. Thompson, P., Hayashi, K., Sowell, E., Gogtay, N., Giedd, J., Rapoport, J., de Zubicaray, G., Janke, A., Rose, S., Semple, J., Doddrell, D., Wang, Y., van Erp, T., Cannon, T., Toga, A.: NeuroImage 23, S2-S18 (2004)

10. Lui, L.M., Wang, Y., Chan, T.F., Thompson, P.: IEEE (CVPR), New York 2, 1784-1792 (2006)

11. Thiruvenkadam, S., Groisser, D., Chen, Y.: VLSM (2005) 